\title{
2020 expert consensus statement on neuro-protection after cardiac arrest in China
}

\author{
Qingbian Ma ${ }^{1 \#}$, Liqun Feng ${ }^{2 \#}$, Tao Wang ${ }^{3}$, Yongqiu $\mathrm{Li}^{4}$, Zhenzhong $\mathrm{Li}^{5}$, Bin Zhao ${ }^{6}$ Xiuchuan Qin ${ }^{7}$, \\ Qingxi $\mathrm{Li}^{8}$, Shizheng $\mathrm{Wu}^{9}$, Hongbin $\mathrm{Sun}^{10}$, Jun Yuan ${ }^{11}$, Lan Chu ${ }^{12}$, Jian $\mathrm{Wu}^{13}$, Yuxiang Gu ${ }^{14}$, Peter Pang ${ }^{15}$, \\ Zhi Chen ${ }^{16}$, Dongsheng Fan ${ }^{17}$; on behalf of the Brain and Vascular Branch \& First Aid and Resuscitation \\ Branch of the Chinese Society of Cardiothoracic and Vascular Anaesthesiology
}

${ }^{1}$ Emergency Department, Peking University Third Hospital, Beijing, China; ${ }^{2}$ Neurology Department, Anzhen Hospital, Capital Medical University, Beijing, China; ${ }^{3}$ Neurosurgery Department, Peking University Third Hospital, Beijing, China; ${ }^{4}$ Neurology Department, Tangshan Gongren Hospital, Tangshan, China; ${ }^{5}$ Neurology Department, The Second Hospital of Hebei Medical University, Shijiazhuang, China; ${ }^{6}$ Emergency Department, Beijing Jishuitan Hospital, Beijing, China; ${ }^{7}$ Emergency Department, Anzhen Hospital, Capital Medical University, Beijing, China;

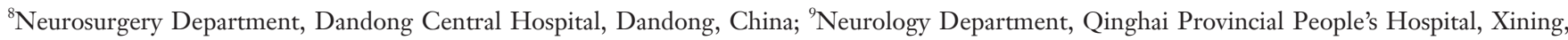
China; ${ }^{10}$ Neurology Department, Sichuan Provincial People's Hospital, Chengdu, China; ${ }^{11}$ Neurology Department, Inner Mongolia People's Hospital, Hohhot, China; ${ }^{12}$ Neurology Department, The Affiliated Hospital of Guizhou Medical University, Guiyang, China; ${ }^{13}$ Neurology Department, Beijing Tsinghua Changgung Hospital, Beijing, China; ${ }^{14}$ Neurosurgery Department, Fudan University Huashan Hospital, Shanghai, China; ${ }^{15}$ Accident and Emergency Department, Yan Chai Hospital, Hong Kong, China; ${ }^{16}$ Beijing Emergency Medical Center, Beijing, China; ${ }^{17}$ Neurology Department, Peking University Third Hospital, Beijing, China

\#These authors contributed equally to this work.

Correspondence to: Professor Dongsheng Fan. Department of Neurology, Peking University Third Hospital, No. 49, North Garden Road, Haidian District, Beijing 100191, China. Email: dsfan2010@aliyun.com; Zhi Chen. Beijing Emergency Medical Center (120), No. 103, Qian Men Xi Da Jie Xicheng District, Beijing 100031, China. Email: cpr120@163.com.

Submitted Nov 06, 2020. Accepted for publication Dec 29, 2020.

doi: $10.21037 /$ atm-20-7853

View this article at: http://dx.doi.org/10.21037/atm-20-7853

\section{Introduction}

Cardiac arrest (CA) is one of the leading causes of human death and disability worldwide. Out-of-hospital CA (OHCA) occurs annually in 420,000 patients in USA, 350,000 in Europe and 544,000 in China $(1,2)$. The incidence rate of OHCA in China is 41.84 per 100,000 (3) and higher when in-hospital CAs are recruited. Although new guidelines have greatly improved the administration of cardiopulmonary resuscitation (CPR), the outcomes of CA have not changed accordingly $(1,4)$. The discharge rate of CA patients is 9\% in Europe and 6\% in North America, but only $2 \%$ in Asia $(\mathrm{P}<0.001)(5)$ and the situation in China is even worse. Research data shows that approximately $70 \%$ of patients with return of spontaneous circulation (ROSC) die from brain damage (6) because the brain is sensitive to ischaemia and hypoxia. After ROSC, any brain injury may lead to residual neurological complications, vegetative conditions, and death.

Clinical studies of CA patients face the difficulties of recruitment and prognosis prediction. This has resulted in a lack of standardized data on brain protection after CPR which is problem for medical practitioners and researchers worldwide. Furthermore, the research needed to produce such data involves comprehensive and complex collaborative treatment and evaluation processes involving multiple scientific and clinical disciplines and requires the participation of a full multidisciplinary team. There is both poor awareness and low implementation of neuroprotection after successful CPR in China and there are currently no guidelines for neuro-protection in CA patients in China or abroad. The Brain and Vascular Branch and the First Aid and Resuscitation Branch of the Chinese Society of Cardiothoracic and Vascular Anaesthesiology invite experts from neurology, neurosurgery, first aid, intensive care, anaesthesiology, cardiovascular science, and pharmacy to establish consensus on neuro-protection after CA.

Target users include pre-hospital emergency medical systems, emergency departments, intensive care units (ICU) 
and related parties. Recruited patients include those who are in the stage between successful CPR and the mid-stage of post-CA syndrome (PCAS). We present the following article in accordance with the RIGHT reporting checklist (available at http://dx.doi.org/10.21037/atm-20-7853).

\section{Methods}

A literature search included studies published from 1960 to March 2018 in the PubMed, Embase, ScienceDirect, Wanfang, and China National Knowledge Infrastructure (CNKI) databases. The evidence was then graded, and the opinions and suggestions of experts sought. All recommendations were agreed to by expert vote.

Evidence was graded on the following basis:

(I) clinically relevant results from meta-analyses of large-sample double-blind randomised controlled trials (RCTs) or medium-sample RCTs;

(II) small-sample RCTs, RCTs without blind method, or RCTs employing effective surrogate markers;

(III) non-randomised controlled studies, observational (cohort) studies, case-control studies, or crosssectional studies;

(IV) comments of expert committees or relevant authorities.

The recommendation levels were as follows:

Grade A: data from multiple clinical randomised trials or meta-analyses;

Grade B: data from a single randomised trial or a single non-randomised study;

Grade C: data from expert consensus, case studies or medical standards;

Grade U: data lacking evidence or having insufficient evidence.

\section{Brain injury mechanism after CA}

Although the brain accounts for only $2 \%$ of total body weight, it has an extremely high metabolic rate. The ratio of cerebral blood flow to cardiac output lies between $15 \%$ and $20 \%$ and brain oxygen consumption accounts for $20-25 \%$ of body intake. The oxygen reserve of the brain is low, $65 \%$ of body glucose consumption occurs in the brain (7), and its tolerance to ischaemia and hypoxia is poor. The primary mechanism of brain injury is closely related to the time window of ischaemia and hypoxia caused by the interruption of blood flow after CA and irreversible damage occurs when cerebral blood flow is completely interrupted for more than 5 minutes. Severe brain blood barrier disruption is also strongly associated with poor neurological outcomes(8). After ROSC, the brain experiences reperfusion injury causing secondary injury. Understanding the pathophysiology of brain injury in CA and proactively performing brain protection to alleviate brain damage will improve the survival rate and neurological prognosis.

\section{Hypoxic-ischaemic brain injury (HIBI)}

Current studies suggest that the main factor affecting the prognosis of CA after CPR is HIBI (9). Studies show that HIBI results in $68 \%$ of in-hospital and $23 \%$ of OHCA deaths (10).

After CA, systemic blood flow stops. Ten to fifteen seconds after cerebral blood flow is interrupted, the oxygen storage of brain cells is exhausted and the production of adenosine triphosphate (ATP) ceases, which affects energydependent particle pathways, causing intracellular $\mathrm{Na}+$ accumulation and cell oedema. After 4 to 5 minutes, ATP is depleted, brain cells begin anaerobic metabolism, and lactic acid accumulation in the brain causes brain cell acidosis.

When cerebral blood flow is interrupted, the depolarisation of brain cell membranes causes an influx of $\mathrm{Ca}^{2+}$ which mainly accumulates in the mitochondria. This further inhibits mitochondrial oxidative phosphorylation causing mitochondrial dysfunction, the rapid consumption of ATP in brain cells, the release of excitatory neurotransmitters, the activation of lipases and proteases, and eventually brain cell apoptosis (11). Fundamental studies have confirmed that the release of large amounts of excitatory amino acids during cerebral ischaemia has toxic effects on neural cells, among which glutamate has the highest content, the widest distribution, and the strongest influence. The ischaemic time frame and glutamate concentration are proportional to the resulting level of neural cell damage (12).

After onset of ischemia, inflammatory processes start immediately and evolve through several phases as well (13). Some studies found inhibited inflammation may decrease pathological damage to brain tissue after cardiac arrest $(14,15)$. To date, our understanding of inflammation after global brain ischemia is partly derived from the expanding knowledge on inflammation after focal brain ischemia although differences exist (16).

\section{Ischaemia-reperfusion brain injury}

During CPR, partial cerebral blood flow is restored. 
Increased blood perfusion results in an increase in reactive oxygen species, inflammatory mediators, cytokines, and neutrophils, which can lead to secondary brain cell damage. After ROSC, brain auto-regulation dysfunction can lead to regional differences in cerebral perfusion. This means continuous hypoperfusion occurs in some brain regions and relative hyperperfusion occurs in others. While hypoperfusion can lead to persistent ischaemic neuronal damage, hyperperfusion can exacerbate brain oedema and brain damage.

Ischaemia-reperfusion injury is primarily caused by oxygen free radical-induced damage cascades, lipid peroxidation, and deoxyribonucleic acid/ribonucleic acid cleavage. This further exacerbates the extent of damage because of changes to blood-brain barrier permeability, complement activation, and the aggregation and adhesion of coagulation factors, platelets, activated microglia, and infiltrated lymphocytes (17). Histological evidence of these damage mechanisms can appear as neuronal necrosis and apoptosis in the hippocampus, cortex, cerebellum, striatum, and thalamus and can be seen hours to days after resuscitation.

\section{Neuro-protection}

Brain protection refers to procedures to reduce brain cell damage and restore neurological dysfunction after brain ischaemia or hypoxia. Current intervention procedures include mild hypothermia therapy, mitochondrial protection, scavenging free radicals, improving cerebral haemodynamics, anti-inflammation and anti-oxidation treatments, and comprehensive medical treatments.

\section{Mild hypothermia therapy}

Mild hypothermia therapy refers specifically to intravascular mild hypothermia, or blood cooling, which is cooling through extracorporeal circulation, intravascular heat exchange, or intravenous infusion. A 1958 study was the first to evaluate its effects in patients following CA and reported the rescue rate of patients treated with low temperature of $33{ }^{\circ} \mathrm{C}$ was $50 \%$ in comparison to $14 \%$ in those treated with normal temperatures (18). In 2002, the $N$ Engl 7 Med published the results of Australian and European prospective clinical studies which confirmed that mild hypothermia therapy can significantly improve the neurological function and survival rate of $\operatorname{OHCA}(19,20)$. Its role in brain resuscitation has quickly remained hot research topic and the recommendation that a body temperature of $32-36{ }^{\circ} \mathrm{C}$ be maintained in patients who are comatose after resuscitation has been made by the International Liaison committee on Resuscitation (ILCOR) and included in CPR guidelines (21). The "Practice Guideline: Reducing Brain Injury After Cardiopulmonary Resuscitation", publication of the American Academy of Neurology in 2017, makes a class A recommendation for mild hypothermia treatment (target temperature of $32-34{ }^{\circ} \mathrm{C}$, maintained for 24 hours) (22).

Brain metabolism can be reduced by $6-10 \%$ for every $1{ }^{\circ} \mathrm{C}$ decrease in body temperature. Mild hypothermia reduces the rate of free radical production, the influx of calcium ions, the degree of mitochondrial damage (23), and the production and release of excitatory amino acids such as glutamate, and inhibits the endogenous and exogenous apoptotic pathway to reduce cell death. It can also limit secondary cell damage by reducing brain oedema, repairing the blood-brain barrier, and inhibiting inflammatory responses (24). The mortality rate may increase by as much as $20 \%$ for each one-hour delay of mild hypothermia therapy (25). Studies showed targeted temperature management can improve long-term neurological outcome, such as memory function (26).

At present, most hospitals in China do not have equipment for direct intravascular blood cooling and instead use other cooling procedures including body cavity, body surface and drug cooling. Body cavity cooling involves the use of cooled sterile saline to lavage and cool the chest or abdominal cavity. However, this may entail operational difficulties such as ice water directly contacting the heart causing ventricular fibrillation or other heart rhythm disorders. Body surface cooling is performed using an ice cap or ice bag placed on the head and the superficial parts of large blood vessels. The procedure is simple, but the cooling speed is slow and the target body temperature is difficult to maintain. Artificial hibernation can also be achieved by administering chlorpromazine, promethazine, or Dilantin.

Although therapeutic hypothermia treatment is currently the only clinically proven brain resuscitation method, more evidence-based studies are required as there are many unresolved issues surrounding its use in clinical practice. Consensus on its treatment length, time course, cooling method, and rewarming speed is lacking and its influence on basal heart rate and neural function remains unclear. Controversy regarding its efficacy and safety in patients with in-hospital PCAS and the complications of its use also exist. 
Recommendation 1: commence mild hypothermia therapy as soon as possible within 12 hours of the cardiopulmonary resuscitation of CA patients (target body temperature of $32-34{ }^{\circ} \mathrm{C}$ for $12-24$ hours). (I, A).

Recommendation 2: intravascular cooling is preferred. If the conditions cannot be satisfied, other cooling methods may be considered. (IV, C).

\section{Drug treatment}

There is presently little direct evidence from clinical studies in the field of drug treatment, due to the urgent condition of CA patients, unpredictability of emergent events, and because many procedures during the rescue process cannot be interrupted. However, some drugs have been clinically shown to protect the brain by safeguarding mitochondria, guaranteeing perfusion, enhancing microcirculation, and improving energy metabolism.

The ROSC in CA patients enables the body to develop rapidly from "the ischaemia and hypoxia state of systemic blood flow disruption" to the "pathophysiological state of systemic ischaemia-reperfusion injury". The process of ischaemia-reperfusion injury at this stage was defined as PCAS by the International Liaison Commission on Resuscitation (ILCOR), the American Heart Association's Emergency Cardiovascular Care Committee (AHAECCC), and the Council on Cardiovascular Surgery and Anaesthesia (CVSA). This process can be divided into four main stages (27).

The first stage is within 20 minutes after CPR has reached ROSC and is defined as the ultra-early stage of PCAS. Here, the oxygen and glucose reserves of brain cells are gradually depleted, mitochondrial functions are damaged, and $\mathrm{Ca}^{2+}$ overload causes brain cell damage. This stage is the key time window for brain protection and mitochondrial protective drugs should be used as soon as possible.

The second stage is from 20 minutes after ROSC to 6-12 hours after resuscitation and is defined as the early stage of PCAS. The restoration of blood flow now results in secondary injury by inflammatory mediators, cytokines, and oxygen free radicals. Protection of mitochondria should continue and treatments providing anti-inflammation, antioxidation, and the elimination of inflammatory factors and oxygen free radicals should be proactively performed.

The third stage from 6-12 to 72 hours after resuscitation is defined as the mid-stage of PCAS and is the main stage of comprehensive treatments to correct metabolic disorders.
The fourth stage commences 3 days after resuscitation and is defined as the recovery stage of PCAS. This stage is the time in which to evaluate brain and neural functions to obtain an accurate prognosis.

\section{Butylphthalide}

Butylphthalide improves cerebral ischaemia, enhances the activity of mitochondrial ATP enzyme and mitochondrial complex IV, protects the integrity of membrane structure and the functions of mitochondria, and improves energy metabolism $(28,29)$. After CA, the endurance effect of ATP promoted by butylphthalide can effectively increase the level and utilization rate of ATP in brain cells and reduce brain damage. In animal study, butylphthalide reduces neurovascular inflammation and ischemic brain injury (30). It can significantly improve the prognosis of the early or recovery stage of cerebral ischaemia through improving microcirculation in the ischaemic area, increasing cerebral blood flow and cerebral blood vessel density, opening collateral circulation, and promoting the recovery of cerebral blood flow (31-34). During the stage of ischaemiareperfusion injury, the anti-radical action of butylphthalide can enhance the activity of superoxide dismutase (SOD) in serum and reduce malondialdehyde (MDA) content to promote the recovery of neurological function. The antiinflammatory and anti-apoptosis effect of butylphthalide may also play an important role in brain protection after CA (35-39). Moreover, butylphthalide can upregulate the expression of 5-hydroxytryptamine (5-HT) in the ischaemic hippocampal penumbra and play a protective role in hippocampal neurons. By reducing the expression of matrix metalloproteinase-9 (MMP-9), butylphthalide also alleviates the degree of blood-brain barrier injury $(40,41)$. Although this research evidence is mainly derived from animal studies of butylphthalide injection in the treatment of ischaemic stroke and phase III multi-centre RCTs $(42,43)$, the mechanisms are sufficiently understood to recommend the use of butylphthalide injection in CA patients after CPR.

Recommendation 3: after establishing effective circulation in pre-hospital first aid CPR, CA patients should immediately receive intravenous butylphthalide infusion. This should be repeated 6 hours later, after which butylphthalide should be administered twice per day, at $25 \mathrm{mg}(100 \mathrm{~mL})$ per dose, for 14 days. (I, B).

\section{Free radical scavengers}

Edaravone is a small-molecule free radical scavenger that effectively passes through the blood-brain barrier, 
Table 1 Recommendations for brain protection after cardiopulmonary resuscitation in cardiac arrest patients

\begin{tabular}{lll}
\hline Treatment & Ultra-early stage (immediately after ROSC) & Early stage (6-12 hours after ROSC) \\
\hline Preferred treatments & Intravascular mild hypothermia therapy & Butylphthalide \\
& Butylphthalide & Edaravone \\
Alternative treatments* & Other cooling procedures & \\
\hline
\end{tabular}

*, alternative treatments are options when the preferred treatment is not available. ROSC, return of spontaneous circulation.

inhibits the activities of xanthine oxidase and hypoxanthine oxidase, stimulates the production of prostacyclin, reduces the production of leukotriene, and directly reduce the concentration of hydroxyl radicals generated after cerebral ischaemia. In this way, it can alleviate the damage caused by free radicals to macromolecules such as lipids, proteins, and nucleic acids and preserve the integrity of the membrane structure of important organelles, such as the cell membrane, mitochondria, and endoplasmic reticulum. The scavenging of free radicals also inhibits the release of inflammatory mediators, and the mitigation of inflammatory responses reduces the delayed death of neural cells, which contributes to the recovery of neural function after cerebral resuscitation $(44,45)$. Fundamental studies have proven that edaravone can significantly improve neural function injuries in mice with brain oedema and in mice treated with CPR to improve survival rates (46).

Recommendation 4: CA patients should commence 30 $\mathrm{mg}$ of edaravone twice per day within 12 hours of CPR and this should continue for 14 days. (II, B).

\section{Calcium channel antagonists}

Calcium channel antagonists can reduce $\mathrm{Ca}^{2+}$ overload in brain cells, relax vascular smooth muscle, resist oxidation, and limit the damage caused by oxygen free radicals to the endothelium. Common calcium channel antagonists include nimodipine and lidoflazine. Although in theory, reducing $\mathrm{Ca}^{2+}$ influx can alleviate brain tissue damage, there is insufficient evidence in current studies demonstrating calcium channel antagonists have brain protection effects and the safety of these antagonists after CPR in CA patients remains to be confirmed.

\section{Excitatory amino acid receptor antagonists}

When cerebral ischaemia occurs, large amounts of glutamate are released, the reuptake of glutamate by neurons is inhibited, and extracellular glutamate accumulates directly causing toxicity and damage to neurons (47). While excitatory amino acid receptor antagonists may be beneficial for brain protection, their use cannot be supported as there is insufficient evidence of their clinical benefit.

\section{Other medicines}

The Practice Guideline: Reducing Brain Injury After Cardiopulmonary Resuscitation released by the American Academy of Neurology in 2017 made a class C recommendation for the use of co-enzyme Q10 to improve the survival rate of out-of-hospital PCAS patients on the basis of mild hypothermia therapy. However, there is insufficient evidence to support or deny the use of steroids to improve survival rates or neurological prognoses (24).

Recommendation 5: evidence for the efficacy and safety of calcium channel antagonists, excitatory amino acid receptor antagonists and steroids, is lacking. (IV, $\mathrm{U}$ ).

\section{Comprehensive medical treatments}

Complications including cerebral oedema and acidosis are seen in CA patients following CPR. Comprehensive medical treatment should be targeted to the specific complication and may include dehydration, anticonvulsant and acid-reducing treatment, glucocorticoid application, oxygen therapy, and blood sugar management (Table 1).

\section{Neurological assessment}

Procedures to monitor, assess, and predict neurological outcomes are important and the accurate estimation of prognosis can guide follow-up treatment. Neurological assessments should be carried out throughout the entire therapeutic process. In particular, the diagnosis of brain death should be made with extreme caution and must be carried out in accordance with current national standards and technical specifications.

After ROSC in hospital, $80 \%$ of patients experience coma and $40 \%$ enter a persistent vegetative state (48). Timely and continuous neurological assessment and effective prediction 
Table 2 Recommended neurological assessments after cardiopulmonary resuscitation in CA patients

\begin{tabular}{lll}
\hline Institution & Early stage (6-12 hours after ROSC) & Mid stage (72 hours after ROSC) \\
\hline Primary hospitals & The GCS & The GCS, clinical symptoms, and signs \\
Hospitals with advanced capabilities & Imaging, biomarkers (NSE, S-100ß) & Imaging, neurophysiology (EEG, SSEP), \\
& & biomarkers (NSE, S-100 3 )
\end{tabular}

CA, cardiac arrest; ROSC, return of spontaneous circulation; GCS, Glasgow Coma Scale; NSE, neuron-specific enolase; EEG, electroencephalogram.

of the prognosis of brain function is required. The definition of a poor prognosis of brain function after CPR includes death, unconsciousness 1 month after CPR, severe disability 6 months after CPR, and the inability of a patient to care for themselves (49). Accurate and reliable indexes must be employed to assess the prognosis and the accuracy and reliability of a poor prognosis are mainly assessed by the false-positive rate (FPR) which should ideally be $0 \%$ (50). A detailed medical history can help predicting the prognosis and should include general conditions and comorbidities (age $>70$ years, history of stroke, renal failure or congestive heart failure before admission), the time and speed of CA onset, and CPR-related conditions (hypoxia time, CPR duration, arrhythmia type). However, these factors are not completely sufficient and reliable and the accuracy of most current clinical research evidence on the neurological prognoses after CA is unsatisfactory due to diagnostic bias. The FPR of prognosis assessment using any single method is high (50) and at present, there is no single examination that can $100 \%$ accurately predict the recovery of neurological function after CA. On this basis, the use of multiple models to evaluate the neurological prognosis of brain resuscitation is recommended. A detailed clinical physical examination remains essential for assessing the prognosis of coma patients after CA. In guidelines for the neurological examination of critically ill patients, the European Society of Intensive Care Medicine proposed that the pupillary light reflex, corneal reflex, and motor responses should be performed in coma patients following CA (51). Somatosensory evoked potential (SSEP) and electroencephalogram (EEG), which have high specificity, can improve the accuracy of the assessment (Table 2).

\section{The Glasgow Coma Scale (GCS)}

The GCS is commonly used for assessing the neurological status of patients. The maximum of 15 points indicates clear consciousness, a score of 8 or less indicates coma status, and the minimum is 3 points. A lower score indicates a more serious disturbance of consciousness. Although the GCS is suitable for the evaluation of neurological function after CPR in CA patients, it contains three aspects; blinking, language, and motor reactions, that are susceptible to factors such as tracheal intubation/incision, facial oedema, sedative muscle relaxation and mild hypothermia treatment. The European Resuscitation Council Guidelines for Resuscitation 2014 recommends the use of the Glasgow Coma ScaleMotor Component (GCS-M) score, which uses the motor responses of the GCS to assess brain function prognosis. Although a GCS-M score of 2 or less suggests a poor neurological function prognosis (50), it is important to be aware that analgesic drugs and muscle relaxants may influence this. The GCS and GCS-M are not specific for CA patients after CPR and do not include pupil size, light response, eye movement and other brainstem responses or the observation of vital signs, which are critical for evaluating neural system function. It is necessary to develop corresponding scales to fill this gap.

\section{Biomarkers}

The advantages of biomarker evaluation are its noninvasiveness and potential for early implementation. Compared with electrophysiology and clinical physical examinations, biomarker evaluation can be quantitative and independent of sedative effects. However, it is difficult to define uniform thresholds for biomarkers and the concentrations of biochemical indicators are constantly changing, limiting their clinical applications.

Neuron-specific enolase (NSE) is released by apoptotic neurons. A previous study (52) showed that an NSE concentration greater than $33 \mu \mathrm{g} / \mathrm{L}$ within $1-3$ days after ROSC in CA patients suggests a poor prognosis. However, in patients receiving hypothermia therapy, the neurological prognosis assessment of CA patients using NSE may result in false positives and false negatives $(53,54)$, suggesting the 
previously used NSE threshold is not a reliable indicator of neurological prognosis. The formation of a prognosis using only one threshold value is not recommended. Early-stage haemodynamics and red blood cell lysis at low temperatures may cause interference with NSE, although serial changes of NSE concentration within $72 \mathrm{~h}$ may be more accurate (55).

Serum S-100 $\beta$ protein is released by glial cells after craniocerebral injury and can also be released by extracranial tissues such as fat and muscle. Some studies suggest that $\mathrm{S}-100 \beta$ can be used as a marker of brain tissue damage. However, the critical levels of S-100 $\beta$ at which a poor prognosis is predicted differ widely among studies, thus its clinical prognostic value awaits further clarification.

\section{Imaging examinations}

Imaging examinations are also important methods for the prognostic evaluation of brain function in CA patients. Conventional brain imaging examinations such as computed tomography (CT) and magnetic resonance imaging (MRI) are less sensitive in detecting the pathological changes of transient systemic hypoxia-ischaemia because neuron damage in this state does not involve the destruction of neuron structures. A recent study used a standardized method to detect differences between white matter and grey matter to reflect severe global brain cytotoxic oedema (56). By detecting the grey to white matter ratio (GWR) in the putamen and internal capsule, the specificity of predicting poor neurological prognosis in patients within 1-7 days after CA was $100 \%$, and the sensitivity reached $44 \%$. Some studies show that advanced MRI sequences such as the apparent diffusivity coefficient (ADC), diffusion tensor imaging, and resting state functional magnetic resonance imaging (fMRI) (57-61) can be used for prognostic evaluation after CA. Although the results of these clinical studies are very promising, further validation is required before they can be routinely used in the clinical setting.

\section{Neurophysiology}

EEG is non-invasive, affordable, can monitor brain electrical activity in real time, and is routinely used for evaluating coma patients. In coma patients after CA, low background activity, status epilepticus, burst suppression and low voltages of EEG indicate poor prognosis (49).

SSEP can reflect functional brain status to some extent, and changes to the primary cortical response (N20) have the greatest clinical significance. In HIBI, the N20 wave may be delayed or lost, reflecting a loss of nerve conduction from the brainstem or thalamus to the cortex. A clinical study (62) has suggested that bilateral N20 wave loss indicates poor prognosis regardless of whether mild hypothermia therapy is performed and a meta-analysis (63) showed that bilateral N20 wave loss in CA patients with cerebral resuscitation had extremely high specificity for indicating a poor prognosis after mild hypothermia therapy. The best time to evaluate the disappearance of the bilateral N20 wave is during the mild hypothermia state and after rewarming (72 hours after ROSC).

\section{Clinical symptoms and signs}

Physical examination can reveal a series of signs indicating a poor prognosis after CA including the disappearance of the pupillary light and corneal reflexes although sedatives and muscle relaxants may restrict adequate physical examination, especially in patients receiving mild hypothermia therapy. Therefore, examination should be delayed for at least 72 hours after rewarming or the withdrawal of sedatives and analgesic drugs and should be repeated multiple times. While the value of the pupillary light and corneal reflexes immediately after ROSC in prognosis prediction is limited, their absence after 72 hours indicates poor prognosis, with a FPR of $0 \%$. It is possible to perform primary screening for coma patients after CA based on the high sensitivity of pain-stimulated motor responses for poor prognosis (49) (the specificity of the pupillary reflex is superior to the corneal reflex (64), then perform further evaluations using multiple methods. Other physical signs (including myoclonus) are unreliable in predicting poor prognosis and are not recommended for prognosis prediction (65).

\section{Summary}

Neuro-protection is one of the keys to successful CPR and good prognosis in CA patients. Patients with ROSC after CPR should receive both treatment procedures to prevent brain injury in the ultra-early stage of PCAS and effective management during the entire therapeutic process. This consensus recommends early diagnosis, pre-hospital intervention, continuous assessment, and comprehensive treatment for all CA patients after CPR to effectively improve the survival rate and the neurological prognosis of patients through standardized management. Knowledge gaps are evident in neuro-protection after cardiac arrest. 
To determine the efficiency and safety of treatment, more questions need to be addressed.

\section{Acknowledgments}

Funding: None.

\section{Footnote}

Reporting Checklist: The authors have completed the RIGHT reporting checklist. Available at http://dx.doi. org/10.21037/atm-20-7853

Conflicts of Interest: All authors have completed the ICMJE uniform disclosure form (available at http://dx.doi. org/10.21037/atm-20-7853). The authors have no conflicts of interest to declare.

Ethical Statement: The authors are accountable for all aspects of the work in ensuring that questions related to the accuracy or integrity of any part of the work are appropriately investigated and resolved.

Open Access Statement: This is an Open Access article distributed in accordance with the Creative Commons Attribution-NonCommercial-NoDerivs 4.0 International License (CC BY-NC-ND 4.0), which permits the noncommercial replication and distribution of the article with the strict proviso that no changes or edits are made and the original work is properly cited (including links to both the formal publication through the relevant DOI and the license). See: https://creativecommons.org/licenses/by-nc-nd/4.0/.

\section{References}

1. Mozaffarian D, Benjamin EJ, Go AS, et al. Executive summary: heart disease and stroke statistics--2015 update: a report from the American Heart Association. Circulation 2015;131:434-41.

2. Girotra S, Nallamothu BK, Spertus JA, et al. Trends in survival after in-hospital cardiac arrest. $\mathrm{N}$ Engl J Med 2012;367:1912-20.

3. Hua W, Zhang LF, Wu YF, et al. Incidence of sudden cardiac death in China: analysis of 4 regional populations. J Am Coll Cardiol 2009;54:1110-8.

4. McNally B, Robb R, Mehta M, et al. Out-of-hospital cardiac arrest surveillance --- Cardiac Arrest Registry to Enhance Survival (CARES), United States, October
1, 2005--December 31, 2010. MMWR Surveill Summ 2011;60:1-19.

5. Berdowski J, Berg RA, Tijssen JG, et al. Global incidences of out-of-hospital cardiac arrest and survival rates: systematic review of 67 prospective studies. Resuscitation 2010;81:1479-87.

6. Taccone FS, Crippa IA, Dell'Anna AM, et al. Neuroprotective strategies and neuroprognostication after cardiac arrest. Best Pract Res Clin Anaesthesiol 2015;29:451-64.

7. Varvarousis D, Varvarousi G, Iacovidou N, et al. The pathophysiologies of asphyxial vs dysrhythmic cardiac arrest: implications for resuscitation and post-event management. Am J Emerg Med 2015;33:1297-304.

8. Kim HI, Lee IH, Park JS, et al. The Usefulness of Quantitative Analysis of Blood-Brain Barrier Disruption Measured Using Contrast-Enhanced Magnetic Resonance Imaging to Predict Neurological Prognosis in Out-ofHospital Cardiac Arrest Survivors: A Preliminary Study. J Clin Med 2020;9:3013.

9. Fugate JE. Anoxic-Ischemic Brain Injury. Neurol Clin 2017;35:601-11.

10. Laver S, Farrow C, Turner D, et al. Mode of death after admission to an intensive care unit following cardiac arrest. Intensive Care Med 2004;30:2126-8.

11. Sekhon MS, Ainslie PN, Griesdale DE. Clinical pathophysiology of hypoxic ischemic brain injury after cardiac arrest: a "two-hit" model. Critical Care 2017;21:90.

12. Rose ME, Huerbin MB, Melick J, et al. Regulation of interstitial excitatory amino acid concentrations after cortical contusion injury. Brain Res 2002;943:15-22.

13. Sekhon MS, Ainslie PN, Griesdale DE. Clinical pathophysiology of hypoxic ischemic brain injury after cardiac arrest: a "two-hit" model. Crit Care 2017;21:90.

14. Zou X, Xie L, Wang W, et al. FK866 alleviates cerebral pyroptosis and inflammation mediated by Drp1 in a rat cardiopulmonary resuscitation model. Int Immunopharmacol 2020;89:107032.

15. Espinosa-Garcia C, Atif F, Yousuf S, et al. Progesterone Attenuates Stress-Induced NLRP3 Inflammasome Activation and Enhances Autophagy Following Ischemic Brain Injury. Int J Mol Sci 2020;21:3740.

16. Chamorro Á, Meisel A, Planas AM, et al. The immunology of acute stroke. Nat Rev Neurol 2012;8:401-10.

17. Wang XP NJ. Advance in research of therapeutic medicines for cerebral ischemia-reperfusion injury. Chinese Journal of New Drugs 2016,;25:659-63. 
18. Williams GR Jr, Spencer FC. The clinical use of hypothermia following cardiac arrest. Ann Surg 1958;148:462-8.

19. Hypothermia after Cardiac Arrest Study Group. Mild therapeutic hypothermia to improve the neurologic outcome after cardiac arrest. N Engl J Med 2002;346:549-56.

20. Bernard SA, Gray TW, Buist MD, et al. Treatment of comatose survivors of out-of-hospital cardiac arrest with induced hypothermia. N Engl J Med 2002;346:557-63.

21. Callaway CW, Donnino MW, Fink EL, et al. Part 8: post-cardiac arrest care: 2015 American Heart Association guidelines update for cardiopulmonary resuscitation and emergency cardiovascular care. Circulation 2015;132:S465-82.

22. Geocadin RG, Wijdicks E, Armstrong MJ, et al. Practice guideline summary: Reducing brain injury following cardiopulmonary resuscitation: Report of the Guideline Development, Dissemination, and Implementation Subcommittee of the American Academy of Neurology. Neurology 2017;88:2141-9.

23. McGinniss J, Marshall P, Honiden S. Novel uses of targeted temperature management. Clin Chest Med 2015;36:385-400.

24. Yenari MA, Han HS. Neuroprotective mechanisms of hypothermia in brain ischaemia. Nat Rev Neurosci 2012;13:267-78.

25. Mooney MR, Unger BT, Boland LL, et al. Therapeutic hypothermia after out-of-hospital cardiac arrest: evaluation of a regional system to increase access to cooling. Circulation 2011;124:206-14.

26. Evald L, Brønnick K, Duez CHV, et al. Prolonged targeted temperature management reduces memory retrieval deficits six months post-cardiac arrest: A randomised controlled trial. Resuscitation 2019;134:1-9.

27. Neumar RW, Nolan JP, Adrie C, et al. Post-cardiac arrest syndrome: epidemiology, pathophysiology, treatment, and Prognostication a consensus statement from the international liaison committee on resuscitation (American Heart Association, Australian and New Zealand Council on Resuscitation, European Resuscitation Council, Heart and Stroke Foundation of Canada, InterAmerican Heart Foundation, Resuscitation Council of Asia, and the Resuscitation Council of Southern Africa); the American Heart Association Emergency Cardiovascular Care Committee; the Council on Cardiovascular Surgery and Anesthesia; the Council on Cardiopulmonary, Perioperative, and Critical Care; the Council on Clinical
Cardiology; and the Stroke Council. Circulation 2008;118:2452-83.

28. Lei H, Zhao C-Y, Liu D-M, et al. L-3-n-Butylphthalide attenuates $\beta$-amyloid-induced toxicity in neuroblastoma SH-SY5Y cells through regulating mitochondrionmediated apoptosis and MAPK signaling. J Asian Nat Prod Res 2014;16:854-64.

29. Liu RZ, Fan CX, Zhang ZL, et al. Effects of Dl-3-nbutylphthalide on Cerebral Ischemia Infarction in Rat Model by Mass Spectrometry Imaging. Int J Mol Sci 2017;18:2451.

30. Yang CS, Guo A, Li Y, et al. Dl-3-n-butylphthalide Reduces Neurovascular Inflammation and Ischemic Brain Injury in Mice. Aging Dis 2019;10:964-76.

31. Cerebral Blood Flow and Metabolism Branch of the Chinese Stroke Association. Chinese Guidelines for Assessment and Intervention of Cerebral Collateral Circulation in Ischaemic Stroke (2017). Chinese Journal of Internal Medicine 2017;56:460-71.

32. Neurology Branch of the Chinese Medical Association, Cerebrovascular Disease Section of Neurology Branch of the Chinese Medical Association. 2014 Chinese guidelines on the diagnosis and treatment of acute ischaemic stroke. Chinese Journal of Neurology 2015;4:246-57.

33. Committee of experts on the management of post-stroke cognitive disorders, Chinese Stroke Association. Expert consensus on post-stroke cognitive disorder management. Chinese Journal of Stroke 2017;12:519-31.

34. Xiong Z, Lu W, Zhu L, et al. Dl-3-n-Butylphthalide Treatment Enhances Hemodynamics and Ameliorates Memory Deficits in Rats with Chronic Cerebral Hypoperfusion. Front Aging Neurosci 2017;9:238.

35. Li Q, Guo Z, Zhang HZ, et al. Effect of Butylphthalide Injection on MDA and SOD in Chronic Cerebral Ischaemia Rats. Journal of Brain and Nervous Diseases 2008;16:522.

36. Huang K, Liu CX, L ZY. Early application of dual antiplatelet combined with butyl phthalide in the treatment of acute ischemic stroke. Chinese Journal of Clinical Research 2017;30:898-902.

37. Wen XR, Tang M, Qi DS, et al. Butylphthalide Suppresses Neuronal Cells Apoptosis and Inhibits JNK-Caspase3 Signaling Pathway After Brain Ischemia /Reperfusion in Rats. Cell Mol Neurobiol 2016;36:1087-95.

38. Dong M, Cheng SB, Guo YF, et al. The effect of NBP on bcl-2 / bax and bim proteins in oxygen-glucose deprivation model of organotypic brain slices. Journal of Apoplexy and Nervous Diseases 2017;34:16-20. 


\section{Page 10 of 11}

39. O'Neill C. PI3-kinase/Akt/mTOR signaling: impaired on/ off switches in aging, cognitive decline and Alzheimer's disease. Exp Gerontol 2013;48:647-53.

40. Zhou Y, Niu LJ, Qi FM, et al. Effect of 3-n-butylphthalide pretreatment on expression of the HSP70 after brain ischemia/reperfusion. Zhongguo Ying Yong Sheng Li Xue Za Zhi 2015;31:136-40.

41. Ji HR, Kong W, Kong LW, et al. Effect of 3-n-butylphthalide pretreatment on structure and function changes of blood brain barrier in rat model of focal cerebral ischemia-reperfusion injury. Chinese Journal of Pathophysiology 2014;11:2079-83.

42. Cui LY, Liu XQ, Zhu YC, et al. Effects of dl-3Butylphthalide on treatment of acute ischemic stroke with moderate symptoms: a multi-center, randomized, doubleblind, placebo-control trial. Chin J Neurol 2005;38:251-4.

43. Zhu Y, Cui L, Gao S, et al. Safety and efficacy of administration of dl-3-n-butylphthalide for acute ischemic stroke: a phase III, multicenter, randomized, double-blind, double-dummy, active-controlled trial. Chin J Neurol 2014;47:113-8.

44. Li JS, Yang YS. Brain Protection effects of Edaravone, a New Free Radical Scavenger. Journal of International Neurology and Neurosurgery 2006;33:125-8.

45. Zhang XY WL, Fan DS, et al. Combined intravenous recombinant tissue plasminogen activator and edaravone for ultra-acute ischemic stroke. Chinese Journal of Neurology 2008;41:201-3.

46. Qin T, Lei LY, Li N, et al. Edaravone improves survival and neurological outcomes after CPR in a ventricular fibrillation model of rats. Am J Emerg Med 2016;34:1944-9.

47. Ding S, Wang T, Cui W, et al. Photothrombosis ischemia stimulates a sustained astrocytic $\mathrm{Ca} 2+$ signaling in vivo. Glia 2009;57:767-76.

48. Zhang N, Liu JG. Research progress on the influence of thrombolysis on cerebral resuscitation in cardiopulmonary resuscitatio. Chinese Journal of Emergency Medicine 2009;8:891-2.

49. Wijdicks EF, Hijdra A, Young G, et al. Practice parameter: prediction of outcome in comatose survivors after cardiopulmonary resuscitation (an evidence-based review): report of the Quality Standards Subcommittee of the American Academy of Neurology. Neurology 2006;67:203-10.

50. Sandroni C, Cariou A, Cavallaro F, et al. Prognostication in comatose survivors of cardiac arrest: an advisory statement from the European Resuscitation Council

\section{Ma et al. Neuro-protection expert consensus on cardiac arrest}

and the European Society of Intensive Care Medicine. Intensive Care Med 2014;40:1816-31.

51. Sharshar T, Citerio G, Andrews PJ, et al. Neurological examination of critically ill patients: a pragmatic approach. Report of an ESICM expert panel. Intensive Care Med 2014;40:484-95.

52. Zandbergen EGJ, Hijdra A, Koelman JHTM, et al. Prediction of poor outcome within the first 3 days of postanoxic coma. Neurology 2006;66:62-8.

53. Rossetti AO, Carrera E, Oddo M. Early EEG correlates of neuronal injury after brain anoxia. Neurology 2012;78:796-802.

54. Terman SW, Hume B, Meurer WJ, et al. Impact of presenting rhythm on short-and long-term neurological outcome in comatose survivors of cardiac arrest treated with therapeutic hypothermia. Crit Care Med 2014;42:2225-34.

55. Stammet $\mathrm{P}$, Collignon O, Hassager C, et al. NeuronSpecific Enolase as a Predictor of Death or Poor Neurological Outcome After Out-of-Hospital Cardiac Arrest and Targeted Temperature Management at $33^{\circ} \mathrm{C}$ and $36^{\circ} \mathrm{C}$. J Am Coll Cardiol 2015;65:2104-14.

56. Gentsch A, Storm C, Leithner C, et al. Outcome prediction in patients after cardiac arrest: a simplified method for determination of gray-white matter ratio in cranial computed tomography. Clin Neuroradiol 2015;25:49-54.

57. Wijman CA, Mlynash M, Caulfield AF, et al. Prognostic value of brain diffusion-weighted imaging after cardiac arrest. Ann Neurol 2009;65:394-402.

58. Vanhaudenhuyse A, Noirhomme Q, Tshibanda LJ, et al. Default network connectivity reflects the level of consciousness in non-communicative brain-damaged patients. Brain 2010;133:161-71.

59. Luyt CE, Galanaud D, Perlbarg V, et al. Diffusion tensor imaging to predict long-term outcome after cardiac arrest: a bicentric pilot study. Anesthesiology 2012;117:1311-21.

60. Norton L, Hutchison R, Young G, et al. Disruptions of functional connectivity in the default mode network of comatose patients. Neurology 2012;78:175-81.

61. Koenig MA, Holt JL, Ernst T, et al. MRI default mode network connectivity is associated with functional outcome after cardiopulmonary arrest. Neurocrit Care 2014;20:348-57.

62. Rothstein TL. Therapeutic hypothermia and reliability of somatosensory evoked potentials in predicting outcome after cardiopulmonary arrest. Neurocrit Care 2012;17:146-9. 
63. Kamps MJ, Horn J, Oddo M, et al. Prognostication of neurologic outcome in cardiac arrest patients after mild therapeutic hypothermia: a meta-analysis of the current literature. Intensive Care Med 2013;39:1671-82.

64. Samaniego EA, Mlynash M, Caulfield AF, et al. Sedation confounds outcome prediction in cardiac arrest survivors treated with hypothermia. Neurocrit Care 2011;15:113-9.

Cite this article as: Ma Q, Feng L, Wang T, Li Y, Li Z, Zhao B, Qin X, Li Q, Wu S, Sun H, Yuan J, Chu L, Wu J, Gu Y, Pang P, Chen Z, Fan D; on behalf of the Brain and Vascular Branch \& First Aid and Resuscitation Branch of the Chinese Society of Cardiothoracic and Vascular Anaesthesiology. 2020 expert consensus statement on neuro-protection after cardiac arrest in China. Ann Transl Med 2021;9(2):175. doi: 10.21037/atm-207853
65. Field JM, Hazinski MF, Sayre MR, et al. Part 1: executive summary: 2010 American Heart Association guidelines for cardiopulmonary resuscitation and emergency cardiovascular care. Circulation 2010;122:S640-S56.

(English Language Editor: B. Draper) 